\title{
Editorial
}

\section{The Role of Clinical Engineers in Dialysis Therapy in Japan}

\author{
Tomotaka Naramura \\ Department of Medical Technology, Faculty of Medical Science, University of East Asia, Shimonoseki, Japan
}

Dialysis therapy requires the involvement of a wide range of specialist healthcare professionals such as physicians, nurses, clinical laboratory technicians, diagnostic radiology technicians, physical therapists, pharmacists, and dieticians. Also, dialysis therapy as a field requires an extremely broad range of academic knowledge across many related areas, including medicine, physics, chemistry, engineering, nutrition science, microbiology, nursing science, and social welfare science, and we need a firm understanding of both medical science and engineering especially. In Japan, nationally qualified clinical engineers are involved in dialysis treatment working alongside the other medical professionals mentioned above. The role of Japan's clinical engineers is quite different from the role of clinical engineers or biomedical engineers in other countries. Japan's national certification system for clinical engineers was legislated in 1987 and is still therefore relatively new.

The concept of team-based medical care was introduced in open-heart surgery with an artificial cardiopulmonary machine and in dialysis therapy for the first time in Japan. Because physicians and nurses use advanced medical devices for treatment, team-based care began with dialysis technicians being engaged mainly in the op-

\section{KARGER}

๑) 2018 S. Karger AG, Basel

E-Mail karger@karger.com

www.karger.com/bpu eration and management of medical equipment under the responsibility of a physician. At that time, dialysis technicians were not certified, so all medical procedures were performed at the physician's discretion. However, with continuous technological development and increasing numbers and types of medical devices available, engineers who could operate and manage medical equipment became essential in treatment, especially dialysis treatment. Against this background, the national certification system for clinical engineers was established in 1987, to cultivate human resources with expertise in both medicine and engineering. Today, there are approximately 70 universities and vocational colleges that train clinical engineers in Japan, and around 20,000 clinical engineers are employed in hospitals. As of 2017, the number of qualified clinical engineers in Japan stands at 41,533.

In Japan, clinical engineers are qualified to conduct and manage the operation and maintenance of life-support systems under the responsibility of a physician. Besides dialysis treatment, clinical engineers are involved in the medical handling of complex medical equipment, such as artificial cardiopulmonary machines in open-heart surgery, artificial respirators in intensive care, and other equipment required in general surgery, percutaneous 
coronary intervention, and endoscopy. The major difference between Japan and other countries is that Japanese clinical engineers are permitted by law to perform certain medical procedures under the supervision of physicians, in addition to maintaining and managing medical equipment. For example, clinical engineers have a number of responsibilities in dialysis therapy, including the following: preparing the dialysis fluid; performing priming and puncture; measuring blood pressure; monitoring patients during treatment; performing blood return after treatment; and operating, maintaining, repairing, and cleaning and disinfecting the dialysis machines. They are also responsible for maintaining the quality of dialysis fluids, including monitoring electrolytes and osmotic pressure and testing for microorganism/endotoxin contamination. In dialysis therapy in Japan, physicians, nurses, and clinical engineers have established a team-based system for conducting dialysis treatment, cooperating together to take advantage of each other's expertise. Specifically, physicians and clinical engineers often collaborate in projects to improve dialysis machines and develop novel treatment methods, actively publishing the results in papers and presenting at conferences. Clinical engineers involved in di- alysis do not simply assist physicians, they exercise their engineering skills as medical professionals at the bedside, moving far beyond maintaining medical equipment. In this context, the central dialysis fluid delivery system that is peculiar to Japan is widely used in dialysis facilities here, with clinical engineers taking responsibility, as an expert, for system management and dialysis fluid purification management [1-3].

Many of the unique and extremely useful clinical engineering technologies developed by clinical engineers are, unfortunately, used only in Japan at this time and are virtually unknown in other countries. This is largely because clinical engineers have published manuscripts written mostly in Japanese in national journals. However, now, with the help of Karger Publishers, Clinical Engineering joins Critical Care Nephrology, Peritoneal Dialysis, and Hemodialysis as the journal's main topics. Through our new affiliation with Blood Purification, I am delighted that we can introduce clinical engineering technologies used in the field of blood purification to readers. We hope to contribute to further development of the journal and look forward to receiving guidance and support from colleagues and readers.

\section{References}

1 Koda Y, Mineshima M: Advances and advantages in recent central dialysis fluid delivery system. Blood Purif 2009;27(suppl 1):23-27.
Tomo T, Shinoda T: Standardization of water purification in the central dialysis fluid delivery system: validation and parametric method. Blood Purif 2009;27(suppl 1):36-40.
Kawasaki T, Uchino J, Shinoda T, Kawanishi $\mathrm{H}$ : Guidance of technical management of dialysis water and dialysis fluid for the Japan Association for Clinical Engineering Technologists. Blood Purif 2009;27(suppl 1):41-49. 